BULL. AUSTRAL. MATH. SOC.

VOL. 12 (1975), 417-423.

\title{
On the boundedness operator
}

\section{Panayotis Th. Lambrinos}

\begin{abstract}
This paper is a continuation of the study of the boundedness operator $\delta$. By determination of the congruences (that is, collapsings) of the smallest lattice containing $\delta$ and closed under application of $\delta$, a new classification of all topological spaces is obtained according to boundedness criteria.
\end{abstract}

Recently [2, 3], I defined and studied the boundedness operator $\delta: 2^{2^{X}} \rightarrow 2^{2^{X}}$ where $X \neq \emptyset$ and for each $B \subset 2^{X}$, $\delta B=\{S \subset X: H \subset B, H \cup\{S\}$ has finite intersection property implies $\mathrm{nH} \neq \emptyset$ \}.

A subset of a topological space is called bounded [2, 3] if it is contained in some finite union of members of every open cover of the whole space. Let $\gamma B$ be the family of all intersections of (non-empty) families of finite unions of members of $B[1,5], \delta^{\nu}$ is defined inductively by $\delta^{\nu}=\delta\left(\delta^{\nu-1}\right),\left(\gamma^{2}=\gamma\right)$, and multiplication of $\delta$ and $\gamma$ by functional composition.

Then $\delta \gamma B$ is the family of the bounded subsets of the space $(X, T)$ where $B$ is a subbasis for the family $T^{C}$ of the closed sets of it [3, 2]. The study of $\delta$ by analogy to the study of the compactness operator $\rho$ of de Groot, Herrlich, Strecker, Wattel [1, 4, 5] provided [2, 3] the relations

(1) $H \subset B \Rightarrow \delta B \subset \delta H$;

(2) $\delta \gamma=\delta$;

Received 3 February 1975. 
(3) $H \subset Y B \cap \delta B, H$ has finite intersection property implies $n_{H} \neq \emptyset$;

(4) $\gamma B \wedge \delta^{\nu} B \subset \delta^{\nu} B$ where $H \wedge B=\{H \cap B: H \in H, B \in B\}$;

(5) $\delta^{\nu} \wedge \delta^{\nu+1}=\delta^{\nu} \cap \delta^{\nu+1}$;

(6) $\delta B=\delta \gamma\left(B \cup \delta^{2} B\right)$

(7) $\delta \subset \delta^{3}$;

(8) $\delta^{2}=\delta^{4}$;

(9) $\delta^{3} \subset \delta \cup \delta^{2} \Rightarrow \delta=\delta^{3}$;

(10) $\gamma \delta=\delta, A \subset B \in \delta B \Rightarrow A \in \delta B$.

The last two relations give to $\delta$ "nicer" properties than those of $\rho$. Relations of the above type are interesting since a complete classification of all topological spaces according to boundedness criteria has been obtained [2, 3] by the determination of the congruences of the resulting monoid $\left\{\gamma, \delta, \delta^{2}, \delta^{3}\right\}$.

Another classification according to boundedness criteria is obtained here by means of the smallest lattice $L$ (with order induced by containment) containing $\delta$ and closed under application of $\delta$ to the members of $L$. The ideas and techniques are inspired by [1]. We have

(11) $\delta \cap \delta^{2}=\delta^{2} \cap \delta^{3}$;

(12) $\delta \cap \delta^{2}=\delta\left(\delta \cup \delta^{2}\right)=\delta\left(\delta^{2} \cup \delta^{3}\right)$;

(13) $\delta^{2} \cup \delta^{3} \subset \delta\left(\delta \cap \delta^{2}\right)$;

(14) $\delta^{2}\left(\delta \cap \delta^{2}\right)=\delta \cap \delta^{2}$.

Proof of (11). By (7), $\delta \cap \delta^{2} \subset \delta^{2} \cap \delta^{3} \subset \delta^{2}$. Assume that $S \in \delta^{2} B \cap \delta^{3} B, \emptyset \neq H \subset B, H \cup\{S\}$ has finite intersection property. Then, by (4), $H^{*}=H \wedge\{S\} \subset \delta^{2} B \cap \delta^{3} B=\gamma\left(\delta^{2} B\right) \cap \delta\left(\delta^{2} B\right)$ and $H^{*}$ has finite intersection property. It follows by (3) that $\varnothing \neq n H$ and thus, $s \in \delta B$; that is, $\delta^{2} \cap \delta^{3} \subset \delta$. 
Proof of (12). By (1), $\delta\left(\delta^{2} u \delta^{3}\right) \subset \delta\left(\delta \cup \delta^{2}\right) \subset \delta^{2} \cap \delta^{3}$. Let $S \in \delta^{2} B \cap \delta^{3} B, \emptyset \neq H \subset \delta^{2} B \cup \delta^{3} B, H \cup\{S\}$ have finite intersection property. Then, as before, $H^{*}=H \wedge\{S\} \subset \delta^{2} B \cap \delta^{3} B$ and thus $s \in \delta\left(\delta^{2} B \cup \delta^{3} B\right)$.

Proof of (13). $\delta \supset \delta \cap \delta^{2} \subset \delta^{2}$ implies by (1) that $\delta^{2} \subset \delta\left(\delta \cap \delta^{2}\right) \supset \delta^{3}$.

Proof of (14). By (1), (13), and (12), we have $\delta\left(\delta\left(\delta \cap \delta^{2}\right)\right) \subset \delta \cap \delta^{2}$. Conversely, by $(7)$ and $(12)$, $\delta \cap \delta^{2}=\delta\left(\delta \cup \delta^{2}\right) \subset \delta^{2}\left(\delta\left(\delta \cup \delta^{2}\right)\right)=\delta^{2}\left(\delta \cap \delta^{2}\right)$.

The above method of proving the converse of (14) may serve also to obtain a clearer proof of $[1,(15)]$.

By the above relations we get the following lattice $L$ :

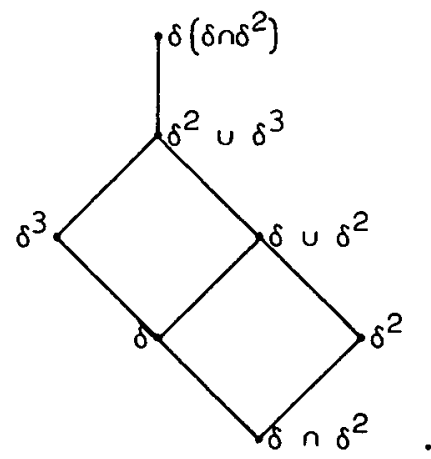

$L$ contains $\delta$ and is closed under application of $\delta$. By Example 1 below, the seven elements of the lattice are in general, distinct. In order to determine all the possible congruence relations (that is, collapsings) of the lattice, the following will be useful.

$$
\begin{aligned}
& \text { (i) } \delta^{2} \subset \delta^{3} \text { or } \delta^{3} \subset \delta^{2} \text { if and only if } \\
& \text { (ii) } \delta \subset \delta^{2} \text { or } \delta^{2} \subset \delta \text { if and only if } \\
& \text { (iii) } \delta\left(\delta \cap \delta^{2}\right)=\delta^{2} \cup \delta^{3} .
\end{aligned}
$$

Proof. (i) $\Rightarrow$ (ii). If $\delta^{2} \subset \delta^{3}$ then, by (11), 
$\delta^{2}=\delta^{2} \cap \delta^{3}=\delta \cap \delta^{2} \subset \delta$ and if $\delta^{3} \subset \delta^{2}$ then, by (7), $\delta \subset \delta^{2}$.

(ii) $\Rightarrow$ (iii). If $\delta \subset \delta^{2}$ then, by (1), $\delta^{3} \subset \delta^{2}$ and thus $\delta\left(\delta \cap \delta^{2}\right)=\delta^{2}=\delta^{2} \cup \delta^{3}$. If $\delta^{2} \subset \delta$ then, by (7), $\delta^{2} \subset \delta^{3}$ and thus $\delta\left(\delta \cap \delta^{2}\right)=\delta^{3}=\delta^{2} \cup \delta^{3}$.

(iii) $\Rightarrow$ (i). If there exist $A \in \delta^{2} B-\delta^{3} B$ and $B \in \delta^{3} B-\delta^{2} B$ then, by (10), $\delta^{2} B \notin A \cup B \leqslant \delta^{3} B$. Nevertheless, by (13), $A \in \delta\left(\delta \cap \delta^{2}\right) \rightarrow B$ and by $(10), A \cup B \in \delta\left(\delta \cap \delta^{2}\right)-\delta^{2} \cup \delta^{3} \neq \emptyset$.

Now, in order to obtain a complete classification of all topological spaces, let 2 denote the class of spaces for which a certain collapsing of $L$ occurs.

If $\delta \notin \delta^{2}$ and $\delta^{2} \notin \delta$ then, by (15), (9), the space belongs either to the class $2_{1}$ : no collapse, or to the class

$$
\mathcal{R}_{2}: \delta\left(\delta \cap \delta^{2}\right) \supset \delta^{2} \cup \delta^{3}=\delta u \delta^{2} \supset \delta^{3}=\delta \supset \delta \cap \delta^{2} \subset \delta^{2} \text {. }
$$

If $\delta \subset \delta^{2}$ then, by (1), (9), $\delta=\delta^{3}$ and thus, by (15), the space belongs to $2_{3}: L=\{\delta\}$ or to

$$
q_{4}: \delta=\delta^{3}=\delta \cap \delta^{2} c \delta^{2}=\delta u \delta^{2}=\delta^{2} u \delta^{3}=\delta\left(\delta \cap \delta^{2}\right) \text {. }
$$

Finally, if $\delta^{2} \subset \delta$ then, by (7), (15), the space belongs to $R_{3}$ or to $R_{5}: \delta^{2}=\delta \cap \delta^{2} \subset \delta=\delta u \delta^{2}=\delta^{3}=\delta^{2} u \delta^{3}=\delta\left(\delta \cap \delta^{2}\right)$ or to $2_{6}: \delta^{2}=\delta \cap \delta^{2} \subset \delta=\delta u \delta^{2} \subset \delta^{3}=\delta^{2} u \delta^{3}=\delta\left(\delta \cap \delta^{2}\right)$.

THEOREM. Every topological space belongs to one of the non-empty disjoint classes $Q_{i}, 1 \leq i \leq 6$.

Proof. By the above mentioned argument, the classes $\eta_{i}, 1 \leq i \leq 6$, exhaust all possibilities for spaces and they are by construction disjoint. Finally, they are also non empty because of the following:

EXAMPLE 1. Let $\left(X_{1}, T_{1}\right)$ be an infinite topological space such that 
the family of its bounded subsets $\delta T_{1}^{c}$ equals the family of the countable subsets of $X_{1}$ (for example, this happens in the space $\Omega_{0}$ of the countable ordinals with the usual order topology [2, Example 5.1.v]). Let also $\left(X_{2}, T_{2}\right)$ be an infinite discrete space and $\left(X_{1} \cup X_{2}, T\right)$ their disjoint topological union. By Theorem 2.4 [2],

$$
\delta T^{c}=\left\{S \subset X_{1} \cup X_{2}: S \cap X_{1} \text { countable and } S \cap X_{2} \text { finite }\right\}
$$

Then it is proved that $\delta^{2} T^{c}=\left\{M \subset X_{1} \cup X_{2}: M \cap X_{1}\right.$ finite $\}$ and $\delta^{3} T^{c}=\left\{N \subset X_{1} \cup X_{2}: N \cap X_{2}\right.$ finite $\}$. It follows that for the space $(X, T), \delta \notin \delta^{2}$, and $\delta^{2} \notin \delta$;

(I) if $X_{1}$ is uncountable then $(X, T)$ is a $2_{1}$-space;

(II) if $X_{1}$ is countable then $(X, T)$ is a $2_{2}$-space;

(III) every finite space is a $2_{3}$-space;

(IV) every infinite discrete space is a 24 -space;

(v) every infinite compact space is a 2 -space;

(VI) $\Omega_{0}$ is a $2_{6}$-space.

With respect to the $S$-classification of $[2,3]$ we get $q_{1} \cup 2_{6}=S_{5}$, $2_{3}=s_{1} \cup s_{2}, 2_{2} \cup 2_{4} \cup 2_{5}=s_{3} \cup s_{4}$.

Using the following relation (16) one can obtain another proof of (6) by modification of methods given in [5].

(16) $\delta(H \cup B)=\delta H \cap \delta B \cap \delta(H \wedge B)$.

Proof. By (1), $H \subset H \cup B \supset B$ and $H \wedge B \subset \gamma(H \cup B)$ imply that $\delta(H \cup B) \subset \delta H \cap \delta B \cap \delta(H \wedge B)$. Conversely, let $S \in \delta H \cap \delta B \cap \delta(H \wedge B), \emptyset \neq D \subset H \cup B$, and $D \cup\{S\}$ have the finite intersection property. If $D_{1}=D \cap H=\emptyset$ or $D_{2}=D \cap B^{\circ}=\varnothing$ then กD $\neq \varnothing$. If $D_{1} \neq \emptyset \neq D_{2}$ then $\emptyset \neq D^{*}=D_{1} \wedge D_{2} \subset H \wedge B$ and $D^{*} \cup\{S\}$ 
has finite intersection property. Therefore $\emptyset \neq n D^{*}=n D$. Since in each case $\emptyset \neq n D$, we conclude that $S \in \delta(H \cup B)$.

Proof of (6). By (2), (16),

$$
\delta_{Y}\left(B \cup \delta^{2} B\right)=\delta\left(B \cup \delta^{2} B\right)=\delta B \cap \delta^{3} B \cap \delta\left(B \wedge \delta^{2} B\right),
$$

and by (7), (1), (4), $\delta B \subset \delta^{3} B \subset \delta\left(B \wedge \delta^{2} B\right)$.

Despite the similarity of the above result to that of [1] there exists no connection between them in that neither constitutes a generalization of the other. However, in the light of (10), some of the following open problems may be easier than the corresponding ones for $p[1,5]$.

(a) Given an operator $f: 2^{2^{X}} \rightarrow 2^{2^{X}}$, zonder what conditions does it coincide with the boundedness operator $\delta$ ? (Boundedness axiomatization problem.)

(b) Determine whether or not $\left\{H \subset 2^{X}: \delta H=B\right\}$ may be empty, as well as conditions to guarantee the existence of a largest $H$ lor $H$ of maximal families).

(c) The corresponding problems for $\delta^{2}$.

(d) Determine the possible relations between $\delta$ and $\rho$ (some are evident).

\section{References}

[1] J. de Groot, H. Herrlich, G.E. Strecker, E. Wattel, "Compactness as an operator", Compositio Math. 21 (1969), 349-375.

[2] Panayotis Lambrinos, "A topological notion of boundedness", Manuscripta Math. 10 (1973), 289-296.

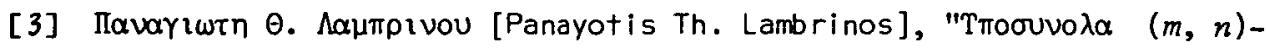

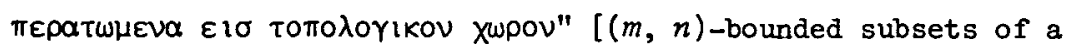
topological space], (Doctoral Dissertation, University of Thessaloniki, Thessaloniki, Greece, 1974 ).

[4] G.E. Strecker, E. Wattel, H. Herrlich, and J. de Groot, "Strengthening Alexander's subbase theorem", Duke Math. J. 35 (1968), 671-676. 
[5] E. Wattel, The compactness operator in set theory and topology (Mathematical Centre Tracts, 21. Mathematisch Centrum, Amsterdam, 1968).

Department of Mathematics,

University of Thessaloniki,

Thessaloniki,

Greece.

Present address:

Mathematical Institute,

University of Oxford,

St Giles,

England. 